\title{
Die neue Prüfungskommission und die Prüfungen 2013 und 2014
}

\section{Urs Strebel}

Präsident der Facharztprüfungskommission AIM von SGAM und SGIM

Ich danke Dr. H. U. Späth, Dr. Bernhard Rindlisbacher, Dr. D. Widmer und Dr. Ch. Berendonk für die kritische Durchsicht des Manuskripts.
Korrespondenz:

Dr. med. Urs Strebel Spital Männedorf Asylstrasse 10 CH-8708 Männedorf
Es ist weder das Ziel noch die Aufgabe dieses Artikels, die Frage zu beantworten, warum und wie die beiden Curricula zusammengelegt wurden. Dies wurde von den beiden Fachgesellschaften SGIM und SGAM und vom Schweizerischen Institut für Weiter- und Fortbildung (SIWF) entschieden (s. diverse Artikel und Briefe in SÄZ und PrimaryCare). Die Fachgesellschaften SGIM und SGAM stehen hinter dem Entscheid, und die Facharztprüfungskommission (FAPK) betrachtet die Lösung als gelungenen Kompromiss.

Als Präsident der FAPK Allgemeine Innere Medizin (AIM) möchte ich in diesem Beitrag aufzeigen, wie die Bildung der gemeinsamen Kommission ablief, welche Ziele sie hat und wie die Prüfungen 2013 und 2014 aussehen werden. Den Entscheid für eine gemeinsame FAPK fällten die Vorstände der SGIM und der SGAM im Frühjahr 2011.

\section{Zusammensetzung der neuen Facharzt- prüfungskommission AIM}

Die Mitglieder werden von beiden Vorständen bestimmt. Aus der 8-köpfigen FAPK der SGIM traten im Hinblick auf die gemeinsame Kommission zwei Mitglieder zurück. Die neue Kommission sollte 10 Mitglieder (je 5 Vertreter von SGIM und SGAM) zählen. Der bisherige Präsident der FAPK SGIM übernahm als 11. Mitglied den Vorsitz. Er hat bei Stimmengleichheit den Stichentscheid, wobei innerhalb der Kommission Konsens herrscht, dass alle wichtigen Entscheide einstimmig gefasst werden müssen. Exofficio-Mitglied mit beratender Stimme ist ein Vertreter des Instituts für Medizinische Lehre (IML) der Universität Bern, das die Prüfungen begleitet und auswertet.

Die FAPK umfasst zurzeit folgende Mitglieder: Präsident: Urs Strebel, Spital Männedorf. Vertreter der SGIM: Jürg Beer, Kantonsspital Baden; Martin Häcki, Zürich; Franco Muggli, Vezia; Christoph Meier, Triemlispital Zürich, und Albert Zingg, Geroldswil. Vertreter der SGAM: Anne Ehle Roussy, Bellevue; Andreas Rothenbühler, Lyss; Monique Savopol, Fribourg; Hansueli Späth, Langnau, und Daniel Widmer, Lausanne. Vertreter des IML: Christoph Berendonk, Bern.

\section{Vorgehensweise}

Am 28.7.2011 traf sich ein Ausschuss von 5 Mitgliedern (2 SGIM, 3 SGAM) in Bern zu einer vorbereitenden Sitzung, und am 19.4.2012 tagte die neue Kom-
2012 war das letzte Jahr, in dem die Schweizerischen Gesellschaften für Allgemeine Innere und für Allgemeine Medizin je eine eigene Facharztprüfung (FAP) durchgeführt haben. Ab 2013 gibt es als indirekte Folge der bilateralen Abkommen mit der EU nur noch eine gemeinsame FAP und einen gemeinsamen Facharzttitel in Allgemeiner Innerer Medizin.

Die Curricula Innere Medizin und Allgemeine Medizin können noch bis Ende 2015 nach dem alten Modus absolviert werden. Ab 2016 existiert nur noch ein gemeinsames Weiterbildungsprogramm, das neben zwei frei wählbaren Jahren drei Jahre Innere Medizin vorschreibt.

mission zum ersten Mal in globo. Es galt, das Vorgehen für die kommenden Jahre festzulegen. Basis war das WBP AIM (www.fmh.ch/bildung-siwf/ weiterbildung_allgemein/weiterbildungsprogramme/ allgemeine_innere_medizin.html). Hauptaufgabe der Kommission ist die Organisation und Durchführung der schriftlichen Prüfung, die folgende Bedingungen erfüllen soll:

- Fragen aus allen Gebieten der Inneren Medizin (siehe oben WBP und Link);

- Fragen, für deren Beantwortung neben dem während der WB erworbenen Wissen auch geeignete Lehrmittel zur Verfügung stehen;

- Fragen in möglichst nur einer Sprache, um die der FAPK SGIM von früher bekannten, mit der Übersetzung verbundenen Probleme zu umgehen;

- Gewährleistung der praktischen Durchführbarkeit, d.h. genügend Fragen und Personal für Korrekturen bei einer geschätzten jährlichen Teilnehmerzahl von 600 Kandidatinnen und Kandidaten.

\section{Bisherige Facharztprüfung Innere Medizin (SGIM)}

Die SGIM stellte in den letzten Jahren an ihrer schriftliche Prüfung mit gut 400 Kandidaten jeweils 120 Multiple-Choice-Fragen (MCQ) aus dem Medical Knowledge Self-Assessment Program (MKSAP) des American College of Physicians (ACP, Amerikanische Internisten-Gesellschaft). Während wir die Fragen bis 2007 in die Landessprachen übersetzten, verzichten wir seit 2008 darauf, weil wir (a) bei zahlreichen Fragen Unterschiede in der Beantwortung in 
Abhängigkeit der Sprache feststellen mussten (Probleme der Übersetzung sind in der Schweiz von eidgenössischen Gesetzen und Verordnungen bekannt, weshalb immer die Sprache der Stammversion angegeben wird) und weil (b) die Prüfungskommission der Ansicht ist, dass heute alle Ärztinnen und Ärzte die englische Sprache wenigstens passiv beherrschen müssen, da viele wichtige Artikel auf Englisch publiziert werden. Die Vorteile der MKSAP-Fragen sind ihre praktische Relevanz (die FAPK achtet bei der Auswahl der Fragen für die FAP darauf, dass die amerikanischen Fragen auch auf unsere Verhältnisse zutreffen), die Korrektheit der Antworten (alle Antworten werden von Spezialisten überprüft) und ihre Praxistauglichkeit (die Fragen werden jährlich von Tausenden amerikanischer Ärztinnen und Ärzte studiert und gelöst). Dazu kommt, dass MC-Fragen computerisiert korrigiert werden können und mit den MKSAP-Heften ein erschwingliches und überschaubares Lehrmittel zur Verfügung steht, für dessen Nutzung wir jedes Jahr das Einverständnis des ACP einholen müssen, das bisher immer gewährt wurde (was leider bei Fragen aus Grossbritannien noch nicht der Fall ist). Die mündliche Prüfung wurde 2009 von der SGIM abgeschafft, da sie einen grossen organisatorischen Aufwand bedeutete, aber in keiner Weise diskriminierte.

\section{Facharztprüfung AIM 2013: Multiple-Choice- und Kurzantwort-Fragen} Die FAP der SGAM bestand bis 2012 aus einem schriftlichen und einem mündlichen Teil (der ab 2013 entfällt). An der schriftlichen Prüfung wurden den Kandidaten ca. 25 Fälle mit Kurzantwortfragen (KAF) vorgelegt. Dabei müssen die Kandidaten anhand von Fallbeispielen Überlegungen zur Differentialdiagnose, zu weiteren Abklärungen, zur Therapie usw. stichwortartig festhalten (Beispiele im Anhang der Guidance). Die FAPK SGAM hat viel Erfahrung mit diesem Fragetyp und einen grossen Fragenpool in deutscher, französischer und italienischer Sprache.

Der wesentliche Unterschied von MC- und KAFragen besteht darin, dass mit den MCQ stärker das passive Wiedererkennen geprüft wird, mit den KAF hingegen eher das aktive Wissen abgefragt werden kann. Einen Nachteil der KAF stellt die komplexere und aufwendigere Korrekturarbeit dar, die bei mehreren Hundert Kandidaten nicht zu unterschätzen ist. Für die FAPK war klar, dass wir die KAF wegen ihrer Vorteile versuchsweise in die FAP integrieren wollten. Wir beschlossen, dass die FAP AIM 2013 (wird wegen der vielen Teilnehmer neu zweimal jährlich durchgeführt: 25. Juni und 29. Oktober 2013, www.aim-info.ch) neben 100 MC-Fragen auch 5 Fälle mit KAF enthalten soll. Dank der grossen Zahl von Kandidaten hoffen wir, die Kernfrage beantworten zu können, ob die Kandidatinnen in den MCund KA-Fragen vergleichbar oder ganz unterschiedlich abschneiden und ob die Kombination der beiden Fragetypen zu einer qualitativen Verbesserung der Prüfung im Bezug auf die Beurteilung der Kandidaten führt.

Die Bestehensgrenze der Prüfungen im Juni und Oktober wird anhand der individuellen Schwierigkeit der einzelnen Prüfungen bestimmt. Die Bestehensgrenze wird im Juni und Oktober deshalb nicht exakt gleich sein. Je nachdem, ob in der Prüfung viele schwierig oder einfach zu beantwortende Fragen gestellt werden (was mittels entsprechender Software bei der Auswertung der Antworten ermittelt wird), wird die Bestehensgrenze tiefer oder höher angesetzt werden. Ausgangspunkt für diese Berechnungen sind die durchschnittlich erreichten Punktzahlen aller an der Prüfung teilnehmenden Kandidatinnen und Kandidaten. Daneben fliessen aber auch statistische Erfahrungswerte von wiederverwendeten Fragen (Ankerfragen) in die Berechnungen mit ein.

\section{Facharztprüfung AIM 2014: Multiple-Choice- und Script-Concordance-Test-Fragen}

An der Sitzung vom 19. April 2012 wurde ein weiterer Vorschlag eines SGAM-Mitgliedes eingebracht. Eine Gruppe um Prof. Jacques Cornuz aus Lausanne beschäftigt sich mit dem sog. Script Concordance Test (SCT), der weniger das Wissen als solches, als mehr die differentialdiagnostischen Überlegungen der Kandidatinnen testet (Stichwort: «from memorizing to reasoning», www.script.edu.es/portal/en/script_en. html). Der SC-Test wurde ursprünglich in Kanada entwickelt. Praktische Erfahrungen wurden bis anhin vor allem im frankophonen Sprachraum gesammelt. Die SCT-Fragen können auf Englisch gestellt werden, da zu ihrer Beantwortung nur das passive Verstehen dieser Sprache nötig ist. Die Antworten können mittels Computer ausgewertet werden.

Da die FAPK bestrebt ist, die Prüfung zu optimieren, und Vorschläge von SGAM- und SGIM-Mitgliedern gleichwertig aufnehmen möchte, hat sich die Kommission entschieden, an der FAP 2014 neben 100 MC-Fragen 21 SCT-Fragen (7 Fallbeispiele mit je 3 Fragen) zu stellen. Details dazu werden rechtzeitig auf der gemeinsamen Website FAP AIM von SGIM und SGAM für die Prüfung 2014 bekanntgegeben.

\section{Schlussbemerkungen}

Die FAPK hofft, Ende 2014 aufgrund der Resultate der Prüfungen von 2013 und 2014 entscheiden zu können, welche die am besten diskriminierende und am einfachsten durchzuführende Prüfungsform der kommenden Jahre sein wird. Basis für die Vorbereitungen auf die FAP bleiben die MKSAP-Hefte. In der Guidance werden weiter «Current Medical Diagnosis and Treatment», «Oxford Textbook of Primary Medical Care» und «Harrison's Principles of Internal Medicine» empfohlen. Genauso wichtig ist aber die während der WB erworbene Erfahrung, weshalb die FAPK nachdrücklich empfiehlt, das Examen frühestens nach zwei, besser nach $2 \frac{1}{2}$ oder drei Jahren WB in Innnerer Medizin zu absolvieren. 